\title{
Editorial
}

\section{Neoadjuvant S-1 and cisplatin for gastric carcinoma: there will be more to come}

\author{
YASUHIRO KODERA \\ Department of Surgery II, Nagoya University Graduate School of Medicine, 65 Tsurumai-cho, Showa-ku, Nagoya 466-8550, Japan
}

Although surgery continues to play a central role in the treatment of gastric carcinoma, an optimal multidisciplinary approach has been under ongoing investigation to improve therapeutic outcomes. At the January 2006 Gastrointestinal Cancers Symposium, sponsored in part by the American Society of Clinical Oncology, debate focused on whether adjuvant therapy should be administered before or after surgery. Apparently, the intention was to compare the survival benefit of neoadjuvant chemotherapy as evidenced by the British MAGIC trial with that of postoperative chemoradiation established in the North American Intergroup 0116 study [1]. An obvious conclusion was that there was currently no way to answer this question, given that no randomized trial to directly compare these two strategies had been performed, but that each strategy clearly had its own theoretical merits and shortcomings. One disadvantage inherent in postoperative adjuvant therapies may be that this strategy can be offered only to a patient fortunate enough to have been treated with a potentially curative resection. This could be a drawback in the West because a greater proportion of gastric cancers in those countries are deemed unresectable at the time of diagnosis. In contrast, adequate chemotherapy given at the right time could downstage a tumor to such an extent that a locally advanced and unresectable cancer might respond and become resectable.

There was one other Western randomized trial besides Intergroup 0116 in which a postoperative intervention significantly prolonged survival [2]. A striking feature observed in these two trials was that survival of patients treated by surgery alone was extremely poor so poor, in fact, that the patients had in effect undergone noncurative surgery. This is not surprising in the case of 0116, however, if one considers the extent

Offprint requests to: Y. Kodera of nodal dissection performed for this predominantly node-positive population, while no detailed data regarding the extent of surgery are offered in the other study, which also was performed for node-positive gastric cancer. On the other hand, in a subset with a relatively high survival rate, an adjuvant therapy may have very little to add to what has been already achieved by surgery. Investigators were astonished and disappointed to find in a Japan Clinical Oncology Group (JCOG) 9206-2 study that an intensive treatment consisting of intraperitoneal cisplatin (CDDP) and postoperative intravenous 5-fluorouracil/CDDP followed by oral UFT given for 12 months against serosa-positive cancer had absolutely no impact on survival [3]. Lack of impact of intraperitoneal CDDP, in addition to poor compliance (less than $40 \%$ of patients tolerated the planned treatment), has been considered at least partially responsible for this result. A more active and aggressive approach could be tolerated only if given prior to surgery, and JCOG investigators are currently more enthusiastic about exploring the benefits of neoadjuvant chemotherapy. A current treatment regimen extensively tested by JCOG in this setting is a combination of CDDP and oral S-1, which has been acclaimed for achieving a response rate above $70 \%$ in a phase II trial [4], and it is the experience with this regimen that Satoh et al. reported in the current issue of Gastric Cancer [5]. Data presented in this retrospective case series was promising, with a 2-year overall survival rate of $91 \%$ for stage III cancer, median survival time of 1.86 years for stage IV cancer, a downstaging rate of $59 \%$, and a favorable toxicity profile with no treatment-related deaths. Based on these results, a phase III trial for stage III gastric cancer and a phase II trial for stage IV cancer have been launched to test a strategy of preoperative S1/CDDP and surgery followed by postoperative S-1.

Past observations with other types of cancer indicate that a regimen with a pathologic complete response (pCR) rate above $10 \%$ should be used to ensure a 
significant survival benefit in a phase III trial testing neoadjuvant chemotherapy. The overwhelming response rate of the S-1/CDDP regimen was attributable in fact to a number of partial responses, with virtually no cases of complete response observed in a phase II trial [4]. Not surprisingly, a pCR was observed in only 1 of 36 resected cases in the current series. Whether this combination actually works well in the neoadjuvant setting remains a matter of concern, therefore, and this issue can be addressed only through phase III trials, such as the forthcoming trial to be conducted by Satoh et al.

Generally speaking, neoadjuvant chemotherapy should best be attempted in candidates for $\mathrm{R} 0$ resection, and cancers with peritoneal deposits have not been considered good targets of this strategy. Surprisingly, peritoneal deposits observed by staging laparoscopy were eliminated by neoadjuvant chemotherapy in 7 of 10 patients in this series. Postoperative regrowth as peritoneal carcinomatosis was observed in 3 of the 7 responders, and the actual clinical benefit of these seemingly transient responses remains to be elucidated. In this respect, the final result of the phase II trial by Satoh et al. testing the current strategy directed against stage IV cancer also is awaited with much interest. If survival of the downstaged patients in this study were found to be satisfactory, whether the patients had peritoneal deposits prior to the treatment would no longer be of concern in general practice. Laparoscopy could then be deferred until after two cycles of chemotherapy, when it could be performed to determine whether the patient should be treated with a subsequent gastrectomy.

Postoperative chemotherapy following R0 resection in this series and in the forthcoming randomized trial obscures the benefit of neoadjuvant chemotherapy per se. An ongoing JCOG phase III study compares patients treated with neoadjuvant S-1/CDDP plus surgery with those treated with surgery alone, and this study will clarify the survival impact of this specific strategy. Surgery alone remains the standard of care in the Japanese Gastric Cancer Treatment Guidelines because, as mentioned previously, convincing data in support of postoperative chemotherapy have not been accumulated for gastric cancer in Japan. It is very often the case in this country that patients with stage III cancer are prescribed oral fluoropyrimidines postoperatively, however, and Satoh et al. apparently conformed to this community standard. Another pivotal phase III study comparing postoperative S-1 with surgery alone for stage II-III patients has completed the accrual of 1000 patients, and we are now looking forward to an interim analysis scheduled for June of this year. In the meantime, we will wait with great interest to see whether the neoadjuvant S-1/CDDP in addition to postoperative S1 has further impact on survival for this high-risk but potentially curable population.

By this time, most readers will be aware that ongoing clinical trials for gastric cancer in Japan are centered around S-1, a novel oral fluoropyrimidine that achieved a response rate by a single agent of more than $40 \%$ in several late phase II trials. Despite its frequent use in Japan, S-1 has not been as popular a treatment in the rest of the world owing to the severe diarrhea that occurred among Western patients. This adverse reaction occurs because tegafur, the toxic component of S-1, is metabolized differently in Western patients than in Japanese patients as a result of polymorphic differences in the CYP2A6 gene. However, optimal dosage of the S$1 / C D D P$ combination has been discreetly exploited in North America, and a subsequent phase II trial showed this combination to be active (overall response rate, 51\%; median survival, 10.9 months) [6]. A randomized comparison of S-1/CDDP and 5FU/CDDP for advanced gastric cancer is now ongoing in the West, and investigators are optimistic that S-1 has great promise as part of a global standard for treatment of advanced gastric cancer in the near future. We are hopeful that by that time, hard evidence regarding the efficacy of S-1 in adjuvant and neoadjuvant settings will be available from numerous clinical trials conducted in the country where the drug was developed.

\section{References}

1. Macdonald JS, Smalley SR, Benedetti J, Hundahl SA, Estes NC, Stemmermann GN, et al. Chemoradiotherapy after surgery compared with surgery alone for adenocarcinoma of the stomach or gastroesophageal junction. N Engl J Med 2001;345:725-30.

2. Neri B, Cini G, Andreoli F, Boffi B, Francesconi D, Mazzanti R, et al. Randomized trial of adjuvant chemotherapy versus control after curative resection for gastric cancer: 5-year follow-up. Br J Cancer 2001;84:878-80.

3. Miyashiro I, Furukawa H, Sasako M, Yamamoto S, Nashimoto A, Nakajima T, et al. No survival benefit with adjuvant chemotherapy for serosa-positive gastric cancer: randomized trial of adjuvant chemotherapy with cisplatin followed by oral fluorouracil in serosa-positive gastric cancer. Japan Clinical Oncology Group 9206-2. Proceedings of the 2005 Gastrointestinal Cancers Symposium. Hollywood, Florida, January 2005. Alexandria, VA: American Society of Clinical Oncology; 2005.

4. Koizumi W, Tanabe S, Saigenji K, Ohtsu A, Boku N, Nagashima F, et al. Phase I/II study of S-1 combined with cisplatin in patients with advanced gastric cancer. Br J Cancer 2003;89:2207-12.

5. Satoh S, Hasegawa S, Ozaki N, Okabe H, Watanabe G, Nagayama $\mathrm{S}$, et al. Retrospective analysis of 45 consecutive patients with advanced gastric cancer treated with neoadjuvant chemotherapy using an S-1/CDDP combination. Gastric Cancer 2006;9:129-35.

6. Ajani JA, Lee F-C, Singh DA, Haller DG, Lenz H-J, Benson III $\mathrm{AB}$, et al. Multicenter phase II trial of S-1 plus cisplatin in patients with untreated advanced gastric or gastroesophageal junction adenocarcinoma. J Clin Oncol 2006;24:663-7. 